BRAZILIAN JOURNAL

$\mathrm{OF}$

RADIATION SCIENCES

07-03A (2019) 01-09

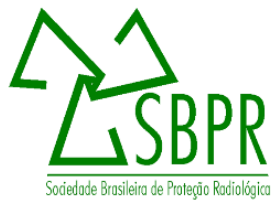

\title{
Control blade history reactivity and pin power effects evaluated with Westinghouse BWR nodal core simulator POLCA8
}

\author{
Guimarães ${ }^{\mathrm{a}}$ P. F. \\ ${ }^{a}$ Westinghouse Electric Sweden AB, SE-721 63, Västerås, Sweden \\ forslupt@westinghouse.com
}

\begin{abstract}
The so-called "Control Blade History" (CBH) effect has always posed a serious challenge for any nodal core simulator in performing Boiling Water Reactor (BWR) core analyses. In this paper a method to handle such CBH effects is proposed based on the idea of interpolating lattice physics data between two extreme cases with regard to $\mathrm{CBH}$, namely, the case with the control rod always inserted during depletion and the case with the control rod never inserted during fuel irradiation. In POLCA8, the latest upgrade of the Westinghouse BWR nodal core simulator POLCA, one applies the methodology to macroscopic cross sections, discontinuity factors, pin powers and detector constants. Overall, the proposed $\mathrm{CBH}$ model performs very well in terms of predictive accuracy of reactivity and pin powers although simultaneous presence of control rods (CRs) and burnable absorbers (BAs) still poses a challenge due to some observed interference of their impact on reactivity. Applying the CBH model for pin power reconstruction is particularly promising and provides excellent prediction accuracy in the vicinity of the CR and at the point of CR withdrawal being the most challenging and critical condition with regard to $\mathrm{CBH}$.
\end{abstract}

Keywords: BWR nodal core analysis, control blade history, nodal cross section models, pin power reconstruction. 


\section{INTRODUCTION}

Historically, the $\mathrm{CBH}$ effect has always posed a serious challenge for any nodal core simulator in performing BWR core analyses and many $\mathrm{CBH}$ models have been proposed in due time to handle this phenomenon (see for example in [1-5]). The difficulty lies in the fact that it is hard to anticipate the exact control rod movements and history during the operation of a BWR. Consequently, the single-assembly lattice physics calculations feeding cross section data to the nodal core simulator normally only consider momentaneous control rod insertion perturbation (i.e. branch) calculations when preparing such nodal cross section data thereby ignoring any account for $\mathrm{CBH}$ effects. Furthermore, the fact that the control rods in BWRs are inserted into inter-assembly gaps between four neighboring fuel elements that are normally filled with water will induce very tilted distributions of power, flux, fuel exposure and nuclide inventories compared to the unrodded situation. In particular, during rodded depletion the increased (thermal) absorption in the control rod in combination with the water displacement effect (i.e. less moderation) will induce a harder local spectrum and an amplified plutonium build-up as well as a reduced burnup rate of uranium in the vicinity of the control rod compared to the unrodded depletion condition. This buildup of local reactivity nearby the control rod will subsequently cause a strong power peaking in the neighborhood of the absorber blades when the control rod is withdrawn from the core due to the locally improved neutron moderation (i.e. by the induced water) and a spectrum softening. Recall that the thermal fission cross section of Pu-239 is 2-3 times larger than the one for U-235 which will enhance this power peaking. Therefore, capturing this $\mathrm{CBH}$ power peaking is of crucial importance due to its direct safety implications (i.e. its impact on thermal margins) and poses a real challenge when considering the conventional two-step methodology applied for nodal core analyses where single-assembly lattice physics transport calculations feed the nodal core simulators with cross section data.

In this work a method to handle such $\mathrm{CBH}$ effects is proposed based on an idea originally published by Højerup and Nonbøl [6]. The main principle of the method is to interpolate lattice physics data between two extreme cases with regard to $\mathrm{CBH}$, namely, the case with the control rod always inserted during depletion and the case with the control rod never inserted during fuel 
irradiation. In POLCA8 [7], the latest upgrade of the Westinghouse BWR nodal core simulator POLCA [8], one applies the methodology to macroscopic cross sections, discontinuity factors, pin powers and detector constants. In this regard, and in order to avoid double counting of the effect of $\mathrm{CBH}$, special care needs to be taken to integrate such a $\mathrm{CBH}$ model appropriately into a nodal code that utilizes a spectrum history correction based on microscopic depletion of important isotopes.

This paper is organized as follows. In Section 2 a short theoretical description of the POLCA8 cross section model and the proposed CBH model is provided. In Section 3 the performance and accuracy of the $\mathrm{CBH}$ model is demonstrated in terms of numerical results for a single-node system. Finally, in Section 4 some concluding remarks are given.

\section{METHODOLOGY}

The cross section model of POLCA8 is based on a formulation where macroscopic cross sections, valid at a so-called "base" condition (i.e. reference depletion condition without any perturbations except for the coolant density), are corrected for by additive correction terms in order to obtain representative cross sections for all anticipated core conditions. Mathematically, based on a Taylor series expansion around the base condition, the cross section model is expressed as

$$
\begin{aligned}
\Sigma\left(E, \rho_{h}, \rho, w_{C B H}, \delta_{C R},\right. & \left.\delta_{S G}, T_{f}, N_{i}, \ldots\right)=\Sigma^{\text {base }}\left(E, \rho_{h}, \rho\right) \\
& +\delta_{C R} \Delta \Sigma^{C R}\left(E, \rho_{h}, \rho, \beta\right)+\delta_{S G} \Delta \Sigma^{S G}\left(E, \rho_{h}, \rho\right) \\
& +d_{D o p}\left(E, \rho_{h}, \rho\right)\left(\sqrt{T_{f}}-\sqrt{T_{f}^{\text {base }}}\right)+c_{B o r}(E, \rho)\left(C_{B}-C_{B}^{\text {base }}\right) \\
& +w_{C B H}\left[\left(1-\delta_{C R}\right) \Delta \Sigma^{C B H, \text { out }}\left(E, \rho_{h}, \rho\right)+\delta_{C R} \Delta \Sigma^{C B H, \text { in }}\left(E, \rho_{h}, \rho\right)\right] \\
& +\sum_{i} \sigma_{i}\left(E, \rho_{h}, \rho, \delta_{C R}, \ldots\right) \Delta N_{i}+\Delta \Sigma^{\text {spat }}+\Delta \Sigma^{\text {het }, \text { byp }} \\
& + \text { cross terms }+ \text { higher order terms }
\end{aligned}
$$

with a similar representation for discontinuity factors, pin powers and detector constants. Here, node average values of the following state parameters are used in the cross section model and in its tabulation: 


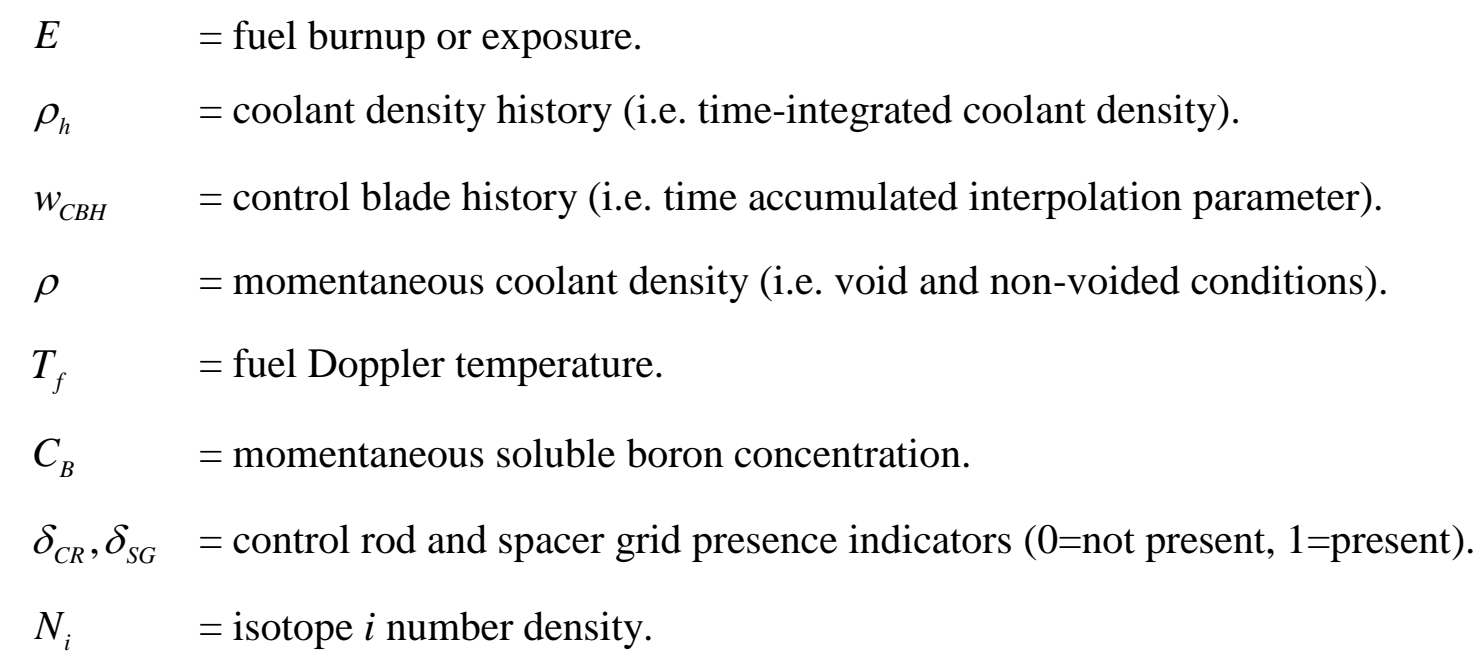

The challenging $\mathrm{CBH}$ effect is handled by interpolating with $w_{C B H}$ in the $\mathrm{CBH}$ correction terms $\Delta \Sigma^{C B H, \text { out }}$ (for unrodded state, $\delta_{C R}=0$ ) and $\Delta \Sigma^{C B H, \text { in }}$ (for rodded state, $\delta_{C R}=1$ ). These $\mathrm{CBH}$ correction terms are computed based on rodded and unrodded lattice depletion calculations to represent the two most extreme $\mathrm{CBH}$ conditions, i.e. depletion history with the $\mathrm{CR}$ always inserted or always withdrawn, respectively.

The fuel exposure dependent $\mathrm{CBH}$ interpolation parameter $w_{C B H}(E)$, i.e. the so-called "control blade history weight factor", is computed for burnup step $n$ as

$$
w_{C B H}\left(E_{n}\right)=\frac{B\left(E_{n}\right)}{T\left(E_{n}\right)} ; \quad w_{C B H}(0)=0, B(0)=0, T(0)=0
$$

where $B\left(E_{n}\right)$ is the so-called "accumulated effective controlled burnup" whereas $T\left(E_{n}\right)$ is the "accumulated effective total burnup". These burnup parameters are recursively updated at each burnup step according to

$$
\left\{\begin{array}{l}
B\left(E_{n}\right)=\left[B\left(E_{n-1}\right)+\delta_{C R} \cdot\left(E_{n}-E_{n-1}\right)\right] e^{-\left(E_{n}-E_{n-1}\right) / E_{\text {decay }}} \\
T\left(E_{n}\right)=\left[T\left(E_{n-1}\right)+\left(E_{n}-E_{n-1}\right)\right] e^{-\left(E_{n}-E_{n-1}\right) / E_{\text {decay }}}
\end{array}\right.
$$


The decay constant $E_{\text {decay }}$ of the exponential in Eq. (3), the so-called "CBH memory", has been empirically determined by back-fitting lattice physics results for various evaluated CBH scenarios. Consequently, by applying this exponential weighting function in the $\mathrm{CBH}$ model, the more recent history events will obtain a higher importance than the older ones.

In POLCA8, an explicit isotopic correction term $\sum_{i} \sigma_{i}\left(N_{i}-N_{i}^{\text {base }}\right)$ based on microscopic depletion of important nuclides is applied to account for the impact of other spectrum history effects besides $\mathrm{CBH}$. Therefore, in order to avoid accounting twice for $\mathrm{CBH}$, the base number density $N_{i}^{\text {base }}\left(E, \rho_{h}, w_{C B H}\right)$ consistent with the base condition in this so-called "residual" history correction needs to be determined based on an interpolation with $w_{C B H}$ according to

$$
N_{i}^{\text {base }}\left(E, \rho_{h}, w_{C B H}\right)=\left(1-w_{C B H}\right) N_{i}^{\text {base }}\left(E, \rho_{h}, w_{C B H}^{\text {const }}=0\right)+w_{C B H} N_{i}^{\text {base }}\left(E, \rho_{h}, w_{C B H}^{\text {const }}=1\right)
$$

Here the unrodded and rodded base number density tables, i.e. $N_{i}^{\text {base }}\left(E, \rho_{h}, w_{C B H}^{\text {const }}=0\right)$ and $N_{i}^{\text {base }}\left(E, \rho_{h}, w_{C B H}^{\text {const }}=1\right)$, are generated internally by POLCA8 consistently with the underlying lattice physics methodology. The interpolation is linear due to the fact that only two conditions are available for this estimation at each burnup point, i.e. the rodded and unrodded number densities. It should also be noted that this interpolation is fully consistent with the linear interpolation performed for the macroscopic cross sections (and other such cross section data) employing the $\mathrm{CBH}$ correction terms $\Delta \Sigma^{C B H, \text { out }}$ and $\Delta \Sigma^{C B H, \text { in }}$ in Eq. (1).

\section{NUMERICAL RESULTS}

All POLCA8 results discussed in this section are based on infinite-lattice single-assembly (i.e. single-node) evaluation of a modern BWR 10x10 fuel design with high enrichment and gadolinium loading (i.e. 4.37 w/o U-235 and $18 \times 8.0$ w/o $\mathrm{Gd}_{2} \mathrm{O}_{3}$ ). Comparisons of POLCA8 results are made 
against an appropriate reference transport solution in order to quantify the model errors. All errors in k-infinity $\left(\varepsilon_{k_{\infty}}\right)$ and pin power of pin $i\left(\varepsilon_{p_{i}}\right)$ are reported as

$$
\left\{\begin{array}{l}
\varepsilon_{k_{\infty}}=\left(k_{\infty}-k_{\infty}^{r e f}\right) \times 10^{5}[\mathrm{pcm}] \\
\varepsilon_{p_{i}}=\left(p_{i}-p_{i}^{r e f}\right) \times 10^{2}[\%]
\end{array}\right.
$$

Absolute errors are chosen for the pin power comparisons in order to not unnecessarily amplify the errors of low power regions and correspondingly not to reduce the errors of high power regions. Recall that relative pin powers are considered here that are normalized to the assembly average value of 1.0 .

Two different types of CR maneuvering strategies typical for real BWR core operation have been considered in this work, namely, 1) a so-called "mono sequence" operation strategy with the control rod inserted next to a two-cycle (or more) burned fuel assembly with the BA burned out, and 2) a so-called "swap sequence" operation strategy with the control rod of a certain CR group inserted next to a fresh (or one-cycle) burned fuel assembly for a short period of time, subsequently to be swapped to another group of control rods, which in turn, after a short irradiation period, again to be swapped to the original group of control rods, etc.. Finally, an extreme case, in which a control rod is present next to a fresh fuel assembly during its first cycle, is also considered.

For k-infinity, three different models have been evaluated; "Kerr_CBH" - representing the bestestimate method combining both the $\mathrm{CBH}$ and the isotopic history correction terms, "Kerr_noCBH" - demonstrating results with the CBH model deactivated thereby only relying on isotopic history tracking, and "Kerr_noCBH.noHis" - representing the most simplistic method with no history model activated. Regarding pin powers, only results for the best estimate method and the most simplistic method are discussed and shown. 
Figure 1: K-infinity error as a function of burnup for both the mono and swap CR maneuvering strategies.

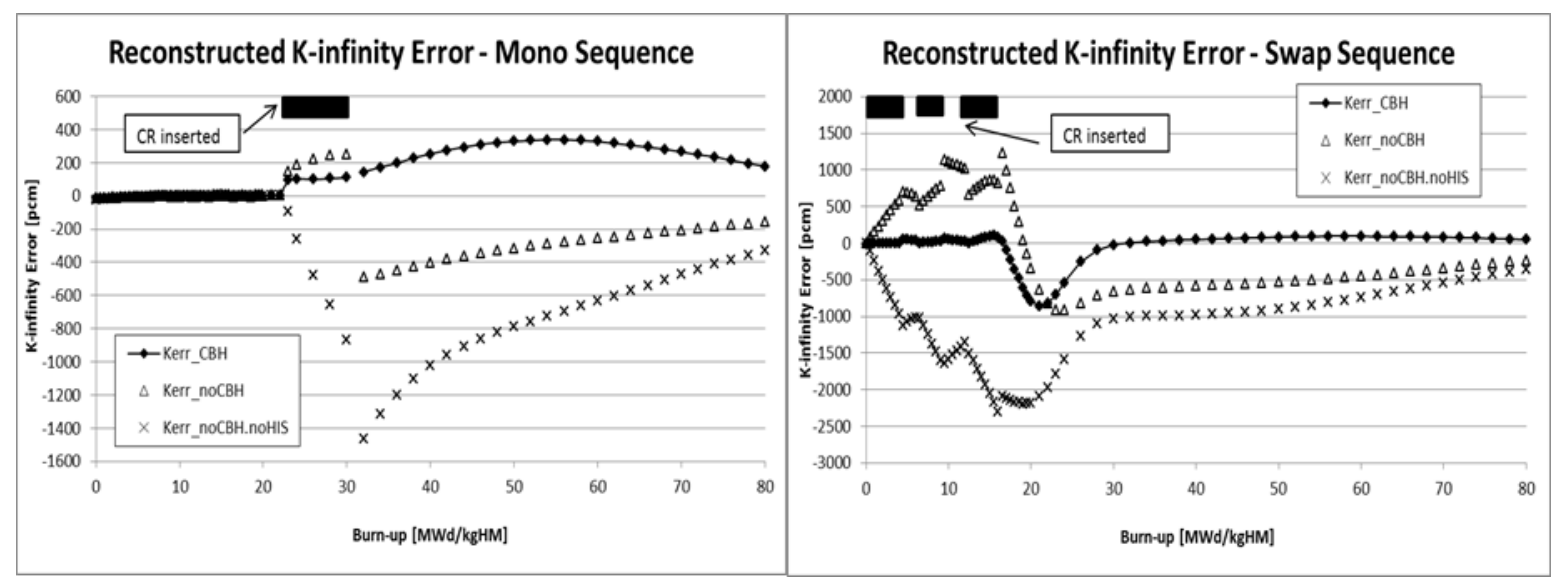

As seen from Fig. 1 for the mono sequence case, the best-estimate method has its largest error around $+340 \mathrm{pcm}$ far beyond the burnup point of CR withdrawal and when the fuel is not driving the reactivity in the core. At the most critical burnup point with regard to $\mathrm{CBH}$, i.e. at the $\mathrm{CR}$ withdrawal point of $30 \mathrm{MWd} / \mathrm{kg}$, the error in k-infinity is less than $+150 \mathrm{pcm}$. This is in contrast to both the isotopic history model and the most simplistic method where both fail to capture this critical burnup point with k-infinity errors as large as $-1500 \mathrm{pcm}$ (i.e. non-conservative).

Considering the swap sequence case in Fig. 1, the best-estimate method shows a very robust behavior in the burnup range of $\mathrm{CR}$ movements (i.e. 0-16 $\mathrm{MWd} / \mathrm{kg}$ ) with all errors below $100 \mathrm{pcm}$ and with no major sensitivity to the underlying CR insertion and withdrawal sequence imposed. In contrast, very large errors up to $-2300 \mathrm{pcm}$ (i.e. non-conservative) are obtained with the most simplistic methods in this same burnup range. 
Figure 2: K-infinity error and the $C B H$ weight factor $w_{C B H}$ as functions of burnup for the fresh CR maneuvering strategy.

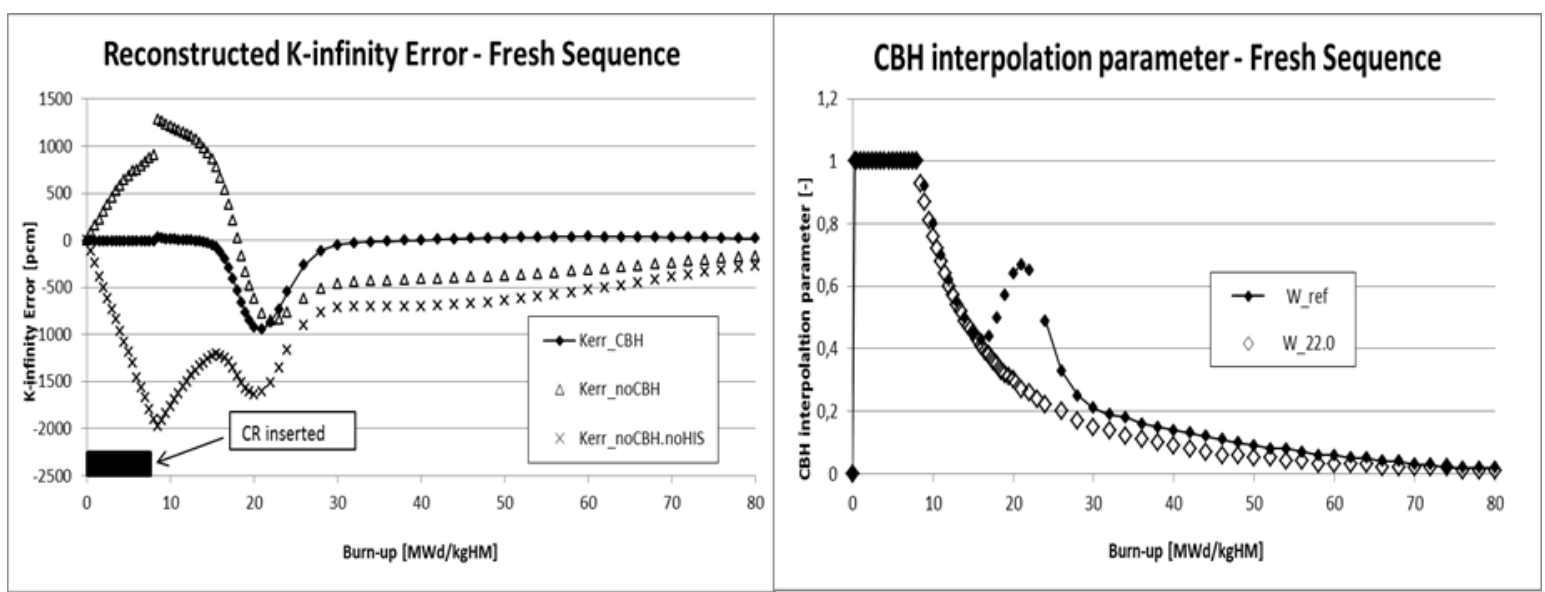

However, at burnups close to the peak k-infinity around $22 \mathrm{MWd} / \mathrm{kg}$, the performance of both the best-estimate and isotopic history tracking method gets deteriorated and an undesired "bubble" behavior in the error function shows up. As seen from Fig. 2, this same bubble behavior in the kinfinity error occurs also for the fresh sequence case.

In Fig. 2, the reference $\mathrm{CBH}$ weight factor $w_{C B H}^{\text {ref }}$ as well as the one computed according to Eq. (2) is shown. It is observed that the reference weight factor starts to decay after the point of CR withdrawal at $8.0 \mathrm{MWd} / \mathrm{kg}$. As expected at high burnups, it approaches asymptotically the value zero representing unrodded base depletion. However, as the fuel BA is successively depleting out, the magnitude of $w_{C B H}^{r e f}$ starts to increase, approaching the rodded base depletion case, until it begins to decline again going towards zero after reaching the k-infinity peak at $22 \mathrm{MWd} / \mathrm{kg}$.

Based on heuristic arguments, this "bubble" behavior of the reference $\mathrm{CBH}$ weight factor is thought to be caused by interference between the inserted CR and the fuel BA. During rodded depletion (i.e. during the so-called $\mathrm{CBH}$ "build-up phase") the flux and power profile will be tilted towards the CR depressing the power density and the burnup locally in this region thereby decreasing the BA depletion rate correspondingly (i.e. causing an asynchronous BA depletion in the 
bundle). Subsequently, after the CR withdrawal (i.e. during the so-called CBH "decay phase") and due to the previous uneven depletion of BA, these BA-pins located close to the CR start to act like a "grey" control rod with its own delayed build-up and decay phase behavior in terms of the spectrum thereby inducing the observed "bubble" behavior. Consequently, in order to capture such a complex burnup behavior, one needs to modify the expression used for computing the CBH weight factor, i.e. Eqs. (2) and (3), to include a more sophisticated correlation of BA depletion into the $\mathrm{CBH}$ model. Note also that this CR and BA interference effect cannot be handled by the isotopic history tracking method, only the $\mathrm{CBH}$ model provides such means.

Table 1: Pin power error statistics for all evaluated CR maneuvering strategies.

\begin{tabular}{cccccccc}
\hline CBH case & $\begin{array}{c}\text { Sample } \\
\text { size }\end{array}$ & $\begin{array}{c}\text { Std } \\
{[\%]}\end{array}$ & $\begin{array}{c}\text { Max } \\
{[\%]}\end{array}$ & $\begin{array}{c}\text { Min } \\
{[\%]}\end{array}$ & $\begin{array}{c}E_{\min } \\
{[\mathrm{MWd} / \mathrm{kg}]}\end{array}$ & $\begin{array}{c}\operatorname{Min}_{\text {noCBH }} \\
{[\%]}\end{array}$ & $\begin{array}{c}\mathrm{E}_{\min , \text { noCBH }} \\
{[\mathrm{MWd} / \mathrm{kg}]}\end{array}$ \\
\hline Fresh & 4884 & 0.84 & 2.81 & -3.68 & 46.0 & $\left.-13.23^{*}\right)$ & $\left.8.0^{* *}\right)$ \\
Mono & 4950 & 0.53 & 3.74 & -2.12 & 54.0 & $-14.37^{*)}$ & $\left.30.0^{* *}\right)$ \\
Swap & 5148 & 0.89 & 3.17 & -4.51 & 48.0 & $-14.65^{*)}$ & $\left.16.0^{* *}\right)$ \\
\hline
\end{tabular}

${ }^{*}$ In the pin closest to the control rod.

${ }^{* * *}$ At the CR withdrawal burnup point.

As demonstrated by Tab. 1 and Fig. 3, very accurate pin powers are obtained with the best estimate method, especially in the vicinity of the CR and at the point of CR withdrawal. Only at very high burnups (see Tab. 1) where the reactivity of the considered fuel segment is becoming very low, the pin power errors become slightly larger with the best-estimate method (i.e. less than $5 \%$ ). In contrast, by disregarding the $\mathrm{CBH}$ effect by deactivating the $\mathrm{CBH}$ and isotopic history tracking models, the pin powers are strongly underestimated by 13-15\% (i.e. negative non-conservative values) close to the $\mathrm{CR}$ and at the point of $\mathrm{CR}$ withdrawal. In other words, neglecting an appropriate modeling of $\mathrm{CBH}$ can potentially have severe safety implications if not properly addressed by other means (e.g. by large design margin penalties). 
Figure 3: Pin power error at CR withdrawal burnup of $8 \mathrm{MWd} / \mathrm{kg}$ for the fresh $C R$ maneuvering strategy. BA-pins are marked with dark grey color.

Best-estimate method ( $\mathrm{CBH}$ and isotopic tracking history):

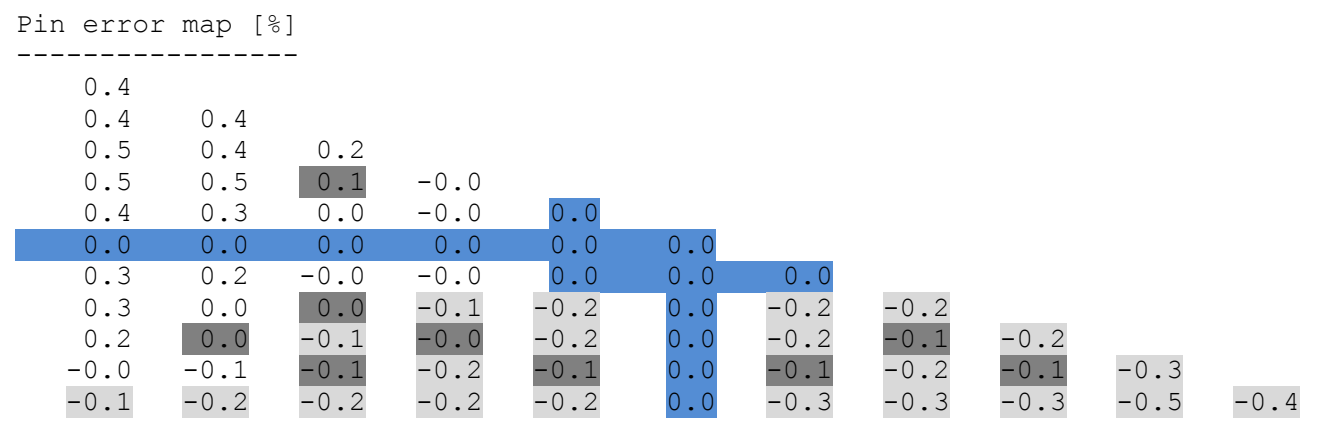

$\underline{\text { Simplistic method (no CBH and no isotopic tracking history): }}$

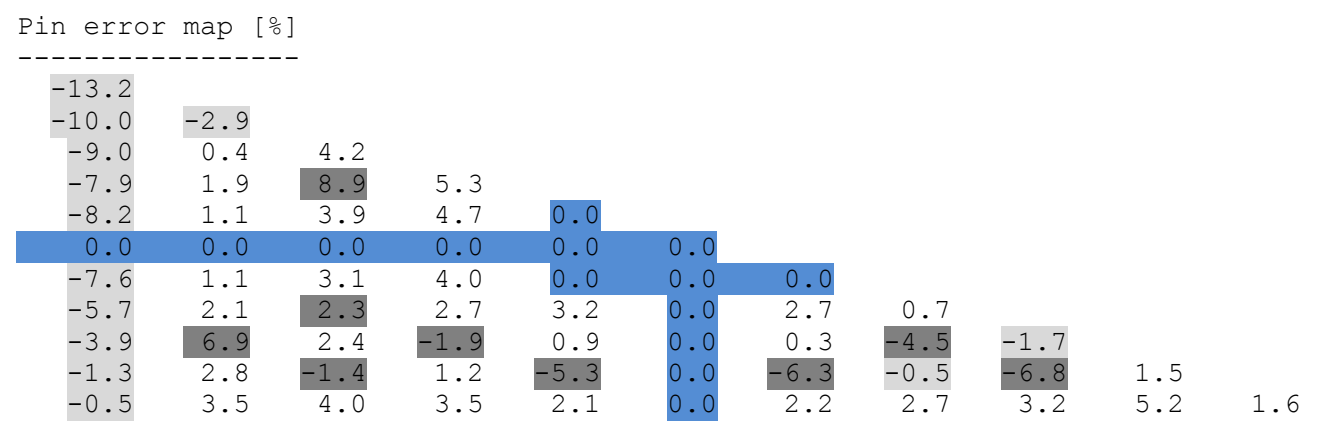




\section{CONCLUSIONS}

In this paper the $\mathrm{CBH}$ effect and its reactor physical implications have been addressed. In particular, a $\mathrm{CBH}$ model to properly handle $\mathrm{CBH}$ effects were implemented in the Westinghouse BWR nodal core simulator POLCA8 and evaluated on simple single-node test cases representing anticipated real core $\mathrm{CBH}$ operation.

It was demonstrated by means of numerical experimentation that the principle of a $\mathrm{CBH}$ model built on interpolation between two extreme cases with regard to $\mathrm{CBH}$ is on a solid basis. It was also shown that the performance of the $\mathrm{CBH}$ model is very robust and shows no major sensitivity to the detailed CR insertion and withdrawal sequence imposed or to the location of BA-pins. In particular, very accurate pin powers are obtained with the $\mathrm{CBH}$ model in the vicinity of the $\mathrm{CR}$ and at the point of $\mathrm{CR}$ withdrawal being the most challenging and critical condition with regard to $\mathrm{CBH}$.

Although the proposed CBH model performs in general very well, the simultaneous presence of CRs and BAs still poses a severe challenge due to some observed interference of their impact on reactivity and therefore needs further attention for its full solution. 


\section{REFERENCES}

[1] IWAMOTO, T.; YAMAMOTO, M. Advanced Nodal Methods of the Few-Group BWR Core Simulator NEREUS. Journal of Nucl. Sci. and Tech., v. 36, no. 11, p. 996-1008, 1999.

[2] IWAMOTO, T.; YAMAMOTO, M. Pin Power Reconstruction Methods of the Few-Group BWR Core Simulator NEREUS. Journal of Nucl. Sci. and Tech., v. 36, no. 12, p. 1141-1152, 1999.

[3] MOON, H. Advanced Core Design Using MICROBURN-B2 BWR Core Simulator. Proc. Am. Nucl. Soc. Topl. Mtg. Advances in Nuclear Fuel Management II, 1997, Myrtle Beach, South Carolina, USA.

[4] FORSLUND, P.; MÜLLER, E.; LINDAHL, S-Ö. Investigation of intranodal depletion effects. Ann. Nucl. Energy, v. 28, p. 225-250, 2001.

[5] BAHADIR, T.; LINDAHL, S-Ö. Evaluation of Rodded BWR Assembly Pin Powers with SIMUlate5. Proc. Int. Conf. Physics of Reactors (PHYSOR 2012), 2012, Knoxville, Tennessee, USA.

[6] HØJERUP, C. F.; NONBØL, E. A Method of Taking Control Rod History into Account in Core Simulation Calculations for BWR's. Proc. Int. Conf. Physics of Reactors (PHYSOR 1990), 1990, Marseille, France.

[7] LIPIEC, W.; FORSLUND GUIMARÃES, P.; JOHANNESSON, S-B.; CASAL, J. Westinghouse BWR Core Analysis Methods Update. Proc. Int. Conf. TopFuel 2015, 2015, Zürich, Switzerland.

[8] LINDAHL, S-Ö.; MÜLLER, E. Z. Status of ABB Atom's Core Simulator POLCA. Proc. Int. Conf. Physics of Reactors (PHYSOR 1996), 1996, Mito, Japan. 